\title{
Enseñanza asistida por pares en una facultad de medicina de Lima, Perú. El caso de la Sociedad Estudiantil de Ciencias Clínicas
}

Peer teaching in a school of medicine in Lima, Peru. The case of the student's society of clinical sciences

Valeria Mariana Li Valverde 1,a;2,b iD, Mario Ezzar Damián Cárdenas 1,a;,, (iD,
Otto Barnaby Guillén-López ${ }^{1, \mathrm{c}}$ (iD)

\section{RESUMEN}

La enseñanza asistida por pares es una metodología educativa donde interactúan grupos de estudiantes. Una parte toma el rol de educador, y la otra toma el rol de alumno. Esto trae varios beneficios a ambas partes. Se presenta el caso de un programa donde se utiliza esta metodología, en el cual estudiantes de una facultad de Medicina en Lima, Perú toman el rol de tutores de estudiantes de años menores de la misma facultad. Se describen también sus actividades y los recursos académicos utilizados.

PALABRAS CLAVE: Educación premédica, enseñanza, facultades de medicina. (Fuente: DeCS BIREME).

\section{SUMMARY}

Peer teaching is an educational methodology in which groups of students interact among each other. One group takes on the role of the educator and the other one takes on the role of the student, which benefits both of them. We report here the case of a program where this methodology was used by students in a medical school in Lima, Peru, who were tutors of junior students from the same faculty. The activities and resources used in this methodology are also described.

KEYWORDS: Premedical education, teaching, medical schools. (Source: MeSH NLM).

Facultad de Medicina, Universidad Peruana Cayetano Heredia. Lima, Perú.

Sociedad Estudiantil de Ciencias Clínicas, Universidad Peruana Cayetano Heredia. Lima, Perú.

Alumno del 6to año de Medicina;

Miembro;

Profesor Auxiliar 


\section{INTRODUCCIÓN}

La enseñanza asistida por pares es una metodología de educación que se caracteriza por la interacción de un grupo de estudiantes en la cual una parte toma el rol de educador, y otra toma el rol de alumno ${ }^{(1)}$. Este método es ampliamente utilizado en educación médica; por ejemplo, en Estados Unidos (EE. UU) se estima que el $50 \%$ de las escuelas de medicina ya han implementado este método de enseñanza dentro de su currícula $^{(2)}$.

Este tipo de enseñanza se divide en dos grandes grupos. Uno es la "enseñanza de par a par" (peer-topeer), que se desarrolla entre miembros del mismo año académico y el otro es la "enseñanza entre pares cercanos" (near-peer), en la cual los docentes son estudiantes de años superiores y otro grupo de estudiantes de años inferiores toma el rol de alumnos. En cualquiera de ellos, existen 3 tipos de actividades: las "mentorías", que se establecen cuando la relación de estudiante: profesor es 1:1 y 2:1; las "tutorías", cuando esta relación es de 3:1 a 10:1; y los "dictados" cuando es mayor a 10:1 ${ }^{(3)}$.

Se reportan beneficios tras su aplicación. En el caso de los tutores, hay una percepción positiva sobre el crecimiento en atributos profesionales y conocimiento (4). Por otro lado, los tutorados aumentan su confianza, reducen el estrés relacionado al estudio y mejoran su rendimiento académico, especialmente alumnos que tienen mayor riesgo de desaprobar ${ }^{(1,5)}$. Asimismo, se genera mayor facilidad para que los estudiantes expresen sus preguntas y mayor aceptación de ellos hacia los tutores que hacia los docentes de facultad, basada en la "congruencia social" ( ${ }^{6}$.

A nivel mundial, especialmente en EE. UU. y en Europa, existe mucha difusión de esta estrategia, con el propósito de potenciar el rendimiento académico de sus estudiantes ${ }^{(5)}$. En Latinoamérica, también se respalda la enseñanza asistida por pares en países como Chile y Colombia ${ }^{(7,8)}$. En Perú, no hay estudios realizados sobre el tema. Apenas un ensayo escrito por Ortega-Miranda E. ${ }^{(9)}$, quien reflexiona que "la mentoría en pares, adecuadamente organizada y supervisada, puede resultar útil y beneficiosa para los estudiantes de reciente ingreso". Mientras otros reportan la escasa participación de los estudiantes como docentes en las facultades de medicina en el Perú, recalcando la nula existencia de estudios sobre este tema en el país, excepto por la existencia de una organización llamada Sociedad Estudiantil de Ciencias Clínicas (SECC) en donde los alumnos toman el rol de profesores hacia estudiantes de años menores ${ }^{(10)}$.

Por ello, decidimos presentar y describir dicho programa educativo que emplea como metodología la enseñanza asistida por pares en una facultad de medicina en Lima, Perú.

\section{PRESENTACIÓN DEL CASO}

La Sociedad Estudiantil de Ciencias Clínicas (SECC) se creó en el año 2015 como una iniciativa de estudiantes de la carrera de medicina de la Universidad Peruana Cayetano Heredia (UPCH) de Lima - Perú, con el objetivo de brindar un apoyo académico a los alumnos de años menores con respecto a temas de los cursos de fisiología y anatomía dictados por la facultad de medicina de dicha universidad.

Actualmente la SECC es una asociación estudiantil sin fines de lucro que tiene como misión el contribuir al desarrollo de la educación médica en el pregrado de medicina a través de la revisión del conocimiento existente e incentivar la generación de nuevo conocimiento sobre temas de interés médico. Para ello, se utiliza la metodología de enseñanza asistida por pares.

Tabla 1. Estrategias didácticas en cada curso.

\begin{tabular}{lcccccc}
\hline CURSO/ESTRATEGIA & $\begin{array}{c}\text { Año de } \\
\text { estudios }\end{array}$ & Tutorías & Repasos & YouTube & Instagram & $\begin{array}{c}\text { Google } \\
\text { drive }\end{array}$ \\
\hline Biología molecular & 2 do & $\mathrm{X}$ & $\mathrm{X}$ & $\mathrm{X}$ & $\mathrm{X}$ & \\
Anatomía & $2 \mathrm{do}$ & $\mathrm{X}$ & $\mathrm{X}$ & $\mathrm{X}$ & $\mathrm{X}$ & \\
Fisiología, embriología e histología & $2 \mathrm{do} / 3 \mathrm{ro}$ & $\mathrm{X}$ & $\mathrm{X}$ & $\mathrm{X}$ & $\mathrm{X}$ & \\
Microbiología & $3 \mathrm{ro}$ & & & $\mathrm{X}$ & & \\
Farmacología & $3 \mathrm{ro}$ & & & $\mathrm{X}$ & & \\
Parasitología & $3 \mathrm{ro}$ & & $\mathrm{X}$ & $\mathrm{X}$ & & $\mathrm{X}$ \\
Semiología & 4 to & & $\mathrm{X}$ & $\mathrm{X}$ & $\mathrm{X}$ & $\mathrm{X}$ \\
\hline
\end{tabular}


Tabla 2. Proceso de postulación de tutores en cada curso.

\begin{tabular}{lcccc}
\hline CURSO/EVALUACIÓN & $\begin{array}{c}\text { Examen } \\
\text { escrito }\end{array}$ & $\begin{array}{c}\text { Exposición de } \\
\text { una clase }\end{array}$ & $\begin{array}{c}\text { Entrevista } \\
\text { personal }\end{array}$ & $\begin{array}{c}\text { Invitación a los miembros } \\
\text { activos de la SECC }\end{array}$ \\
\hline Biología molecular & & $\mathrm{X}$ & & \\
Anatomía & & $\mathrm{X}$ & & \\
Fisiología, embriología e histología & $\mathrm{X}$ & $\mathrm{X}$ & $\mathrm{X}$ & \\
Microbiología & & & $\mathrm{X}$ \\
Farmacología & & & $\mathrm{X}$ \\
Parasitología & & $\mathrm{X}$ \\
Semiología & & & $\mathrm{X}$ \\
\hline
\end{tabular}

Los cursos de la SECC están divididos en tres áreas, abarcando temas de Fisiología, Bioquímica, Anatomía, Farmacología, Microbiología y Semiología. Las áreas se organizan en base a la malla curricular de la Facultad de Medicina de la UPCH ${ }^{(11)}$, de acuerdo a cada año académico (tabla 1). Cada curso está a cargo de un estudiante miembro de la SECC que cumple el rol de "coordinador". Este organiza los horarios y clases a realizar, además de brindar feedback a los tutores.

Se utilizan diferentes estrategias didácticas que varían para cada curso: tutorías, repasos y aprendizaje virtual (tabla 1). Las "tutorías" consisten en brindar clases teóricas de temas que están dentro del sílabo del curso regular de la facultad. Los "repasos" consisten en resolver preguntas teóricas de opción múltiple, uno o dos días antes de que los alumnos tengan sus exámenes parciales y finales oficiales. Estas preguntas son elaboradas por los mismos tutores y el contenido está basado en la tutoría que se ha realizado.

Además, se realiza aprendizaje virtual a través de YouTube $^{\circledR}$, Instagram ${ }^{\circledR}$ y Google Drive ${ }^{\circledR}$. En el canal de YouTube "Sociedad Estudiantil de Ciencias Clínicas UPCH" (12) están disponibles más de 200 videos de temas de los diferentes cursos que se dictan en la SECC. La cuenta en Instagram “@secc. upch" ${ }^{(13)}$, se utiliza para promocionar las actividades de la SECC, y además, permite interactuar con los alumnos a través del planteamiento de casos clínicos y preguntas de diversos cursos. Por último, en Google Drive se almacenan documentos que los tutorados pueden descargar. Dichos documentos generalmente son mapas conceptuales, de máximo dos páginas en formato pdf, sobre temas del curso de semiología.

Los alumnos que son tutores en la SECC, participan de forma voluntaria y son estudiantes de tercero hasta sexto año de la FMAH de la UPCH. Los aspirantes a tutores deben haber aprobado el curso donde deseen participar. Asimismo, deben pasar por un proceso de postulación que es diferente para cada área y curso (tabla 2).

Si bien los tutores no tienen una capacitación universitaria para realizar docencia en medicina, antes del inicio de los cursos del ciclo académico, ellos presencian una clase modelo realizada por uno de los tutores con más experiencia en la SECC. Durante el desarrollo de los cursos, los coordinadores de curso de la SECC tienen el deber de orientar a los tutores para que desarrollen sus actividades de la mejor manera posible. Asimismo, su desempeño es evaluado mediante encuestas a los tutorados luego de cada actividad que se realiza en la SECC. En dichas encuestas se evalúan algunos indicadores en los tutores: manejo de los contenidos del curso, tono de voz, lenguaje claro, capacidad para contestar preguntas de la audiencia, manejo de material de apoyo y estrategias para mantener atenta a la audiencia.

Los tutorados son los alumnos de medicina de la UPCH que acuden a las actividades que ofrece la SECC de manera completamente voluntaria y el único requisito es estar matriculado en la Universidad. El número de tutorados varía de acuerdo a la actividad y al curso. Por lo general, los repasos son las actividades que conglomeran a más tutorados en todos los cursos, superando algunas veces los 100 asistentes. En cambio, las tutorías tienen números variados de asistencia, siendo el promedio de 15 alumnos por sesión.

\section{DISCUSIÓN}

Por lo general, los programas de enseñanza asistida por pares son implementados por la misma universidad para sus propios alumnos, lo que se conoce como 
"institucionalización" o con "coordinación central"; sin embargo, también se reportan programas donde los estudiantes tienen más participación en el desarrollo y organización o incluso, tal como sucede con la SECC, que son dirigidos por los propios estudiantes ${ }^{(14)}$.

Las razones del surgimiento de un programa de enseñanza asistida por pares dependen del contexto específico de cada facultad de medicina. Lo más común es el interés de las instituciones en cultivar en sus estudiantes algunas habilidades en la enseñanza (15), y la necesidad de contar con mayor disponibilidad de personal que realice funciones docentes ${ }^{(4)}$. Otros estímulos, menos frecuentes, provienen de aprovechar los beneficios que genera en los tutores y tutorados, llenar ciertos vacíos en la currícula educativa, o generar un soporte en la preparación para los cursos $(4,14)$.

La SECC ha implementado dentro de su programa, cursos como Anatomía, Bioquímica, Fisiología, Farmacología, Microbiología y Semiología, permitiendo un mayor alcance a los alumnos, por el tipo de cursos y número de actividades que ofrece, así como la oportunidad para los tutores de elegir los cursos a participar. A pesar de que la enseñanza asistida por pares abarca una variedad de tópicos, no es común encontrar programas que se enfoquen en más de un curso ${ }^{(5)}$.

La modalidad de remuneración influye considerablemente en la retención, reclutamiento ${ }^{(16)}$ y motivación de tutores pares. Entre los incentivos intrínsecos están la satisfacción de ayudar a compañeros estudiantes, la ganancia de conocimiento y el desarrollo de habilidades de enseñanza ${ }^{(17)}$. Mientras que el incentivo extrínseco se divide en económico y no económico; este último está representado por créditos académicos, certificados de participación, premios al desempeño, etc ${ }^{(18)}$. La SECC desde sus inicios ha fomentado en sus tutores la satisfacción de ayudar a compañeros de años menores y la oportunidad de crecimiento personal como principales incentivos y motivación para ejercer la labor de tutor. Esto, sumado al factor de ser una organización que no posee ingresos económicos, ha decantado en no emplear estímulos extrínsecos como principal elemento motivador.

A lo largo de sus seis años de vida institucional, el número de tutores postulantes a la SECC ha aumentado, sugiriendo que el incentivo extrínseco no es la principal motivación para ellos. No obstante, para la SECC, la capacitación formal es un reto constante porque no se cuenta con un docente con mejor conocimiento en enseñanza-aprendizaje que asesore a esta organización de manera continua.

Aunque no se ha demostrado objetivamente cuáles serían las habilidades necesarias de los estudiantes para tener éxito en su actividad como tutores ${ }^{(19)}$, es común encontrar entrenamiento formal para ellos ${ }^{(2)}$. Esto está justificado por su falta de experiencia, por tener menos conocimiento del tema, pocas habilidades de enseñanza y el deseo de aumentar su confianza al ejercer la labor de tutor. Aunque no existe un formato de capacitación formal en docencia, ya que difieren en duración y contenido ${ }^{(14)}$, es necesaria la existencia de uno.

Las convocatorias para ser tutores en la SECC son libres al inicio de cada ciclo académico y responden a dos ideas: primero, que todos los alumnos tienen la posibilidad de participar en el programa y, segundo, que el rendimiento académico de un alumno no es discriminatorio para su rol como educador. Wadoodi y Crosby ${ }^{(20)}$, apoyan esto último al sugerir que el desempeño académico no debería ser un impedimento para ser considerado como un tutor, ya que el fin último de esta metodología es que el tutor también pueda nutrirse de los beneficios de participar.

Actualmente existe evidencia que los estudiantes de medicina no son ajenos al uso de redes sociales como herramienta de aprendizaje ${ }^{(21)}$, y que para acceder a estos utilizan los "smartphones", volviéndose una herramienta fundamental para fines educativos, de comunicación y recreación ${ }^{(22)}$. Por ello, la SECC encontró viable involucrar plataformas virtuales para utilizarlas como herramientas de comunicación y de enseñanza.

Todos los tutores y tutorados de la SECC, al ser alumnos de la UPCH, poseen un correo institucional que permite mantener contacto con ellos de manera fluida y formal; mientras que el uso del sistema de almacenamiento Google Drive se utiliza para guardar y compartir archivos, creando un sentido de comunidad que resulta muy eficaz cuando se quiere dar a conocer un mensaje (convocatorias, anuncios, envío de documentos).

El uso de la plataforma de YouTube ${ }^{(12)}$ no ha sido descrito en otros programas de enseñanza asistida por pares. Esta herramienta se utiliza para compartir las clases en formato de videos que, por un lado, facilita que las clases puedan ser revisadas en un número 
infinito de oportunidades y, al mismo tiempo, que puedan ser visualizadas por personas fuera del entorno de la UPCH, incluso del extranjero.

Las plataformas de Facebook ${ }^{(23)}$ e Instagram ${ }^{(13)}$, se usan para brindar información sobre la labor de la SECC, permitir ser ubicada en línea y además, promocionar actividades que se llevan a cabo. Adicionalmente, con Instagram se crea un contenido relacionado con los temas tocados en las tutorías, pero en un formato interactivo, lúdico y didáctico.

Debemos manifestar que el programa que ofrece la SECC aún presenta algunas deficiencias, siendo la principal la falta de capacitación docente formal y constante a los tutores que inician su participación. Actualmente, esta se da en base a experiencias empíricas en la SECC y en las mejoras que se han hecho con las sugerencias de los tutorados en las encuestas.

En conclusión, en nuestro conocimiento, la SECC es el único programa de enseñanza asistida por pares de medicina en el Perú que cuenta con una estructura sólida, que abarca más de un área de enseñanza, cubriendo varios de los cursos de los primeros años de la carrera de medicina y que utiliza varias metodologías de aprendizaje presencial y virtual disponibles.

\section{Contribución de autoría:}

Todos los autores han realizado conjuntamente la argumentación y la redacción del manuscrito.

\section{Declaración de financiamiento y de conflictos de intereses:}

El estudio fue financiado por los autores. Declaran no tener conflictos de intereses.

\section{Correspondencia:}

Valeria Mariana Li Valverde

Correo electrónico: valeria.li.v@upch.pe

\section{REFERENCIAS BIBLIOGRÁFICAS}

1. Ten Cate O, Durning S. Dimensions and psychology of peer teaching in medical education. Med Teach. 2007; 29:546-552. DOI: $10.1080 / 01421590701$ 583816

2. Soriano RP, Blatt B, Coplit L, et al. Teaching medical students how to teach: a national survey of students-as-teachers programs in US medical schools. Acad Med. 2010; 85(11):1725-1731. DOI:10.1097/ ACM.0b013e3181f53273

3. Olaussen A, Reddy P, Irvine S, Williams B. Peerassisted learning: time for nomenclature clarification. Med Educ Online. 2016; 21:30974. DOI: 10.3402/ meo.v21.30974

4. Burgess A, McGregor D, Mellis C. Medical students as peer tutors: a systematic review. BMC Med Educ. 2014; 14:115. DOI: 10.1186/1472-6920-14-115

5. Yu TC, Wilson NC, Singh PP, et al. Medical studentsas-teachers: a systematic review of peer-assisted teaching during medical school. Adv Med Educ Pract. 2011; 2:157-172. DOI: 10.2147/AMEP.S14383

6. Bugaj TJ, Blohm M, Schmid C, et al. Peer-assisted learning (PAL): skills lab tutors' experiences and motivation. BMC Med Educ. 2019;19(1):353. DOI: 10.1186/s12909-019-1760-2

7. Vidal A, Castillo R. Formación de estudiantes de Medicina como tutores pares en aprendizaje basado en problemas. Educación Médica Superior. 2019; 33(3):e1718. (Citado el 04 de octubre del 2020) Disponible en: http://www.ems.sld.cu/index.php/ ems/article/view/1718

8. Torrado-Arenas DM, Manrique-Hernández EF, AyalaPimentel JO. La tutoría entre pares: una estrategia de enseñanza y aprendizaje de histología en la Universidad Industrial de Santander. Medicas UIS. 2016; 29(1):71-75. DOI: 10.18273/revmed. v29n12016008

9. Ortega-Miranda E. Mentoría entre pares en la educación médica de pregrado como herramienta para mejorar el aprendizaje y responder a las demandas de las nuevas generaciones. Acta med peru. 2019; 36(1): 57-61. (Citado el 04 de octubre del 2020) Disponible en: http://www.scielo.org. pe/scielo.php?script=sci_arttext\&pid=S172859172019000100009\&lng=es

10. Chachaima-Mar JE, Ticse R. El estudiante de medicina como profesor en el Perú: un rol importante pero olvidado. Educ Med. 2019. DOI: 10.1016/j. edumed.2019.07.003

11. Facultad de Medicina. Universidad Peruana Cayetano Heredia. Malla Curricular 2020, Facultad de Medicina UPCH. Lima, Perú: Universidad Peruana Cayetano Heredia; 2020 (Citado el 04 de octubre del 2020). Disponible en: https://famed.cayetano.edu.pe/ medicina/malla-curricular

12. Sociedad Estudiantil de ciencias clínicas (SECC). [Internet]. YouTube 2020 [citado 04 de octubre del 2020]. Disponible en: https://www.youtube.com/ channel/UC_gBkRjHBC0OqHX5AbAExcQ

13. Sociedad Estudiantil de ciencias clínicas (SECC). [Internet]. Instagram 2020 [citado 04 de octubre del 2020]. Disponible en: https://www.instagram.com/ secc.upch/ 
14. Herrmann-Werner A, Gramer R, Erschens R, et al. Peer-assisted learning (PAL) in undergraduate medical education: An overview. Z Evid Fortbild Qual Gesundhwes. 2017; 121:74-81. DOI: 10.1016/j. zefq.2017.01.00.

15. General Medical Council. Tomorrow's Doctors: Recommendations on Undergraduate Medical Education. London: General Medical Council; 2003. (Citado el 04 de octubre de 2020). Disponible en: https://www.educacionmedica.net/pdf/documentos/ modelos/tomorrowdoc.pdf

16. Ross MT, Cameron HS: Peer assisted learning: a planning and implementation framework: AMEE Guide no. 30. Med Teach. 2007; 29(6):527-545. DOI: $10.1080 / 01421590701665886$

17. Engels D, Kraus E, Obirei B, et al. Peer teaching beyond the formal medical curriculum. Adv Physiol Educ. 2018; 42(3):439-448. DOI: 10.1152/ advan.00188.2017

18. Buell KG, Pitts W, Edmondson M, et al. Comparing a centralized institutional and student-run peerassisted learning program in medicine. Educ Health. 2018; 31:134-135. DOI:10.4103/efh.EfH_78_18
19. Benè KL, Bergus G. When Learners Become Teachers: A Review of Peer Teaching in Medical Student Education. Fam Med. 2014; 46(10):783-787. (Citado el 04 de octubre del 2020) Disponible en: https://www.stfm.org/FamilyMedicine/ Vol46Issue 10/Bene783

20. Wadoodi A, Crosby JR: Twelve tips for peer-assisted learning: a classic concept revisited. Med Teach. 2002, 24(3):241-244. DOI: 10.1080/01421590220134060

21. Lahiry S, Choudhury S, Chatterjee S, et al. Impact of social media on academic performance and interpersonal relation: A cross-sectional study among students at a tertiary medical center in East India. J Educ Health Promot. 2019; 8:73. DOI: 10.4103/jehp. jehp_365_18

22. Sutherland S, Jalali A. Social media as an openlearning resource in medical education: current perspectives. Adv Med Educ Pract. 2017; 8:369-375. DOI: /10.2147/AMEP.S112594

23. Sociedad Estudiantil de Ciencias clínicas (SECC). [Internet]. Facebook 2020 [citado 04 de octubre del 2020]. Disponible en: https:/www.facebook.com/ SECC.upch

Recibido: 31/10/2020

Aceptado: 16/09/2021 